\title{
Oral Administration of Semicarbazide Limits Weight Gain together with Inhibition of Fat Deposition and of Primary Amine Oxidase Activity in Adipose Tissue
}

\author{
Josep Mercader, ${ }^{1,2}$ Zsuzsa Iffiú-Soltész, ${ }^{1,2}$ Sandy Bour, ${ }^{2}$ and Christian Carpéné ${ }^{1,2}$ \\ ${ }^{1}$ Institut National de la Santé et de la Recherche Médicale, INSERM U1048, Equipe 3, 12MC, IFR 150, \\ Bat. L4, CHU Rangueil, BP 84225, 31432 Toulouse Cedex 4, France \\ ${ }^{2}$ Université Paul Sabatier, 12MC, Centre Hospitalier Universitaire de Rangueil, 31432 Toulouse, France
}

Correspondence should be addressed to Christian Carpéné, christian.carpene@inserm.fr

Received 28 June 2010; Accepted 22 December 2010

Academic Editor: A. Halpern

Copyright (C) 2011 Josep Mercader et al. This is an open access article distributed under the Creative Commons Attribution License, which permits unrestricted use, distribution, and reproduction in any medium, provided the original work is properly cited.

\begin{abstract}
An enzyme hitherto named semicarbazide-sensitive amine oxidase (SSAO), involved in the oxidation of primary amines, is abundantly expressed in adipocytes. Although SSAO physiological functions remain unclear, several molecules inhibiting its activity have been described to limit fat accumulation in preadipocyte cultures or to reduce body weight gain in obese rodents. Here, we studied whether oral administration of semicarbazide, a prototypical SSAO inhibitor, limits fat deposition in mice. Prolonged treatment with semicarbazide at $0.125 \%$ in drinking water limited food and water consumption, hampered weight gain, and deeply impaired fat deposition. The adiposomatic index was reduced by $31 \%$, while body mass was reduced by $15 \%$. Such treatment completely inhibited SSAO, but did not alter MAO activity in white adipose tissue. Consequently, the insulinlike action of the SSAO substrate benzylamine on glucose transport was abolished in adipocytes from semicarbazide-drinking mice, while their insulin sensitivity was not altered. Although semicarbazide is currently considered as a food contaminant with deleterious effects, the SSAO inhibition it induces appears as a novel concept to modulate adipose tissue development, which is promising for antiobesity drug discovery.
\end{abstract}

\section{Introduction}

Pharmacological agents known to inhibit a membrane enzyme involved in the oxidation of various primary amines, and hitherto named semicarbazide-sensitive amine oxidase (SSAO, E.C. 1.4.3.6, pending novel classification E.C. 1.3.4.21) have been reported to limit body weight gain in diverse animal models. Most of these observations of such "slimming effect" with the tested agents were not expected since they were evidenced in studies initially focused on vascular pharmacology. The first reported observation was made by $\mathrm{Yu}$ and coworkers in the obese and diabetic KKAy mice [1]. Whilst the authors aimed to demonstrate an antiatherogenic effect of (E)-2-(4-fluorophenethyl)-3fluoroallylamine (FPFA), they observed that this compound reduced weight gain in obese KKAy mice fed an atherogenic diet. In the same report, $\mathrm{Yu}$ and colleagues demonstrated that FPFA was able to inhibit both SSAO and monoamine oxidases (MAO). More recently, by performing pharmacological research on arterial thickness alteration, Mercier and colleagues repeatedly administrated the reference inhibitor of SSAO, namely, semicarbazide, at $100 \mathrm{mg} / \mathrm{kg}$ bw/ $\mathrm{d}$ to SpragueDawley [2], and Brown-Norway rats [3]. In both models, the authors observed a dramatically reduced body weight gain in response to the SSAO inhibitor, which also induced a decrease in the pressure resistance of arteries, as initially expected.

We have also reported that aminoguanidine, which inhibits SSAO [4] together with nitric oxide synthases (NOS) and diamine oxidase (DAO) [5], was able to limit WAT extension without notably altering calorie intake and body weight gain in obese Zucker rats [6]. Then, we observed that the combined inhibition of SSAO and MAO, obtained by repeated injections of semicarbazide plus pargyline, or 
by daily i.p. administration of phenelzine (an antidepressant which inhibits both MAO and SSAO), produced concomitant limitation of body weight gain in the obese Zucker rat $[7,8]$. Therefore, at least four distinct pharmacological agents tested in vivo to inhibit SSAO were able to alter energy balance and to lower body weight gain in rodents.

SSAO is historically known for its presence in vessels [9]: in endothelial cells, where it is known as SSAO/VAP1 owing to its vascular adhesion properties [10] and in smooth muscle cells, where it is involved together with another copper-containing amine oxidase, the lysyl oxidase, in extracellular matrix maturation $[2,11]$. In fact, SSAO is also highly expressed in white adipose tissue (WAT) [12]. Tissue-distribution studies have recently evidenced that the SSAO amount in adipocytes is extremely elevated, regarding gene expression [13], protein abundance [14], or activity level [6], including in man [15]. With an approach aiming at unravelling the role for such SSAO abundance at the surface of fat cells [15], we observed in vitro that exogenous amines exert insulin mimicry when added to adipocyte preparations. Actually, at submillimolar concentrations, benzylamine elicits, in a SSAO-dependent manner, an activation of glucose transport and an inhibition of lipolysis in isolated fat cells, from human [15] or rodent origin [16]. In addition, benzylamine [17], methylamine [18], or other SSAO substrates [19], activate adipocyte differentiation in several preadipocyte lineages and therefore partly reproduce the adipogenic action of insulin. Lastly, in vitro experiments showed that the hydrazine derivative phenelzine (which inhibits SSAO) alters the adipocyte differentiation of cultured human and mouse preadipocytes [20]. We therefore hypothesized that endogenous or dietary amines may reproduce in vivo such anabolic insulin-like effects, and if the amines can reach WAT, any sustained pharmacological inhibition of their oxidation could hamper fat deposition. In this context, it appeared essential to verify whether inhibition of fat mass extension was an important issue occurring during the body weight gain reduction induced by the above-mentioned agents, all of them sharing the property to inhibit SSAO.

This prompted us to further test whether the per os administration of the prototypical SSAO inhibitor, semicarbazide, was limiting fat deposition. The following results show that, when given in the drinking water, semicarbazide not only inhibited the SSAO activity in WAT, but also limited food consumption, and to a larger extent, hampered fat accretion in both visceral and subcutaneous fat depots. Moreover, the prevention of body weight gain observed during oral administration of semicarbazide was not accompanied by any worsening of the plasma levels of metabolites or oxidative stress markers. Finally, since semicarbazide oral toxicity has been recently suspected [21-23], the need for further investigations of the putative antiobesity effects of other SSAO inhibitors is discussed.

\section{Materials and Methods}

2.1. Animals and Treatments. FVB/n male mice (Charles River, l'Arbresle, France) were separated in two groups of
8 mice with equivalent body weight at the age of 5 weeks. They were housed at 2 animals per cage with unlimited access to standard rodent chow (Global rodent diet, Harlan, France) and water (control), or to a semicarbazide solution (semicarbazide-drinking). Semicarbazide hydrochloride (Sigma-Aldrich, Saint Quentin Fallavier, France) was dissolved in drinking water and given as a $0.125 \%$ solution that was changed weekly. Body mass, food, and water consumption were checked weekly, and at the end of an 8 -week treatment period, the mice were sacrificed after overnight fasting. Ten other 10 -wk-old FVB/n mice grown under standard conditions were used for tissue distribution study of SSAO activity and preliminary lipolytic studies. Housing conditions and experimental procedures followed in the IFR 150 animal unit were in accordance with the European Union regulations on the use of animals for scientific research.

2.2. Tissue Sampling and In Vitro Functional Assays on Adipocytes. Once obtained, plasma was immediately frozen at $-80^{\circ} \mathrm{C}$, and circulating metabolites (glucose, insulin, triglycerides, fatty acids, etc.) were determined using a Cobas-Mira + multi-analyser, according to the manufacturer' instructions (Roche, Neuilly, France). Once weighed, the perirenal, retroperitoneal, and epididymal white adipose tissues were pooled (and named as visceral WAT), immediately digested to obtain adipocyte preparations for glucose uptake assays and lipolysis measurements, as previously described [8], or for cell size determination under microscope using Lucia G software (Nikon). Portion of the tissues were also frozen for further determinations of DNA and protein contents and for amine oxidase assays. DNA content was assessed in WAT after proteinase $\mathrm{K}$ digestion, chloroform/ethanol extraction, and 260/280 nm spectrophotometric readings. Protein content was determined using DC Protein Assay kit (BioRad, Hercules, CA).

2.3. In Vitro 2-Deoxyglucose Uptake Assay. Freshly isolated adipocytes were diluted in around 10-fold their volume of Krebs-Ringer containing $15 \mathrm{mM}$ sodium bicarbonate, $10 \mathrm{mM}$ HEPES, $2 \mathrm{mM}$ pyruvate, and $3.5 \%$ serum bovine albumin. Then $400 \mu \mathrm{L}$ of cell suspension was distributed into plastic incubation vials and incubated $45 \mathrm{~min}$ at $37^{\circ} \mathrm{C}$ with the tested agents, just before $10 \mathrm{~min}$ exposure to $0.1 \mathrm{mM}\left[{ }^{3} \mathrm{H}\right]$-2-deoxyglucose (2-DG). Separation between extracellular and internalized hexose was performed on $200 \mu \mathrm{L}$ aliquots by centrifugation through dinonyl-phthalate layer which allowed to separate buoyant intact fat cells from medium, as previously described [6]. 2-DG uptake was expressed as fold increase over basal uptake or even as percentage of maximal response to insulin, with basal uptake set at 1 or at $0 \%$, respectively [8].

2.4. Amine Oxidase Activity. Oxidative deamination was measured by extracting the oxidation products of $0.1 \mathrm{mM}$ $\left[{ }^{14} \mathrm{C}\right]$-benzylamine (from Amersham Biosciences, Buckinghamshire, UK) or $0.5 \mathrm{mM}\left[{ }^{14} \mathrm{C}\right]$-tyramine (from SigmaAldrich, St Quentin Fallavier, France) after incubation for $30 \mathrm{~min}$ in $200 \mu \mathrm{L}$ of $200 \mathrm{mM}$ phosphate buffer, $\mathrm{pH} 7.5$, in the 
presence of protease inhibitors and approx. $100 \mu \mathrm{g}$ protein of homogenates prepared just before assays from thawed tissues, as previously described [24]. 15-min preincubation with $1 \mathrm{mM}$ semicarbazide or $0.5 \mathrm{mM}$ pargyline was used to selectively inhibit SSAO or MAO activity, respectively, as previously reported [25].

2.5. Gene Expression Analysis. Real-time quantitative RTPCR was performed using oligonucleotide primers specific for the indicated genes, designed with Primer Express software (Perkin-Elmer Life Sciences, Courtaboeuf, F), and the sequences of which are reported in [25], for MAO$A, M A O-B, A O C 2$, and $A O C 3$, or in [26] for adipokines, or either given as supplemental data to [27]. Total RNAs were extracted from mature adipocytes, using RNeasy minikit, then reverse-transcribed using random hexamers and Superscript II reverse transcriptase (Invitrogen, CergyPontoise, F). Reactions without reverse transcriptase (RT-) were performed in parallel to estimate DNA contamination. Real-time RT-PCR was performed using the primers in the presence of $6.25 \mathrm{ng}$ cDNA and SYBR green Universal PCR Mix (Eurogentec, Angers, F). Fluorescence was analysed in an ABI PRISM 7500 Sequence Detection device (Taqman, Applied Biosystems, Foster City, CA). Analysis of $18 \mathrm{~S}$ RNA was performed in parallel using the Ribosomal RNA Control Taqman Assay Kit (Applied Biosystems) to normalize gene expression as already reported [25, 26]. For each gene, results were expressed as arbitrary units: $2^{\text {(Ct18S-Ctgene) }} \times(1-$ $\left.1 /\left(2^{\text {Ct RT-Ctgene }}\right)\right) \times 10^{6}$, where Ct corresponds to the number of cycles reaching fluorescence threshold.

2.6. Statistical Analysis. Data are given as means \pm standard error of the mean (SEM). Comparisons between semicarbazide-treated and control groups were determined using an unpaired Student's $t$-test.

\section{Results}

3.1. Tissue Distribution of SSAO Activity in Mice. Table 1 shows that the SSAO activity was higher in adipose depots than in any other studied organ. As previously reported [28], the oxidation of the prototypic SSAO substrate benzylamine was inhibited by semicarbazide in all the homogenates. However, the visceral WAT of $\mathrm{FVB} / \mathrm{n}$ mouse was richer in SSAO activity than the subcutaneous WAT and than the interscapular brown fat. Aorta, duodenum, and kidney exhibited a lower capacity to oxidize benzylamine than the adipose depots.

\subsection{Changes in Body Weight and Adiposity in Semicarbazide-} Drinking Mice. FVB/n mice exhibits a rapid growth after weaning, which accounts for a week increase of about $10 \%$ of their body mass. We have therefore tested whether semicarbazide oral treatment could hamper adipose tissue development during such period of intense anabolism and substantial fattening. To this aim, semicarbazide was administered at $125 \mathrm{mg} / 100 \mathrm{~mL}$ in the drinking water of 5week-old mice. Since changes in body mass and in water
TABLE 1: Tissue distribution of semicarbazide-sensitive amine oxidase activity in control mice.

\begin{tabular}{lc}
\hline Tissue & $\begin{array}{c}\text { Semicarbazide-inhibited oxidation } \\
\text { of } 0.1 \mathrm{mM} \text { benzylamine } \\
\text { (nmoles/mg protein/min) }\end{array}$ \\
\hline Visceral WAT & $4.402 \pm 0.368(8)$ \\
Subcutaneous WAT & $2.250 \pm 0.144(4)$ \\
Interscapular brown & $0.860 \pm 0.027(4)$ \\
fat & $0.506 \pm 0.064(10)$ \\
Aorta & $0.201 \pm 0.018(4)$ \\
Duodenum & $0.018 \pm 0.005(10)$ \\
\hline
\end{tabular}

Homogenates were preincubated for $15 \mathrm{~min}$ without or with $1 \mathrm{mM}$ semicarbazide before a 30 -minute incubation in the presence of $0.1 \mathrm{mM}\left[{ }^{14} \mathrm{C}\right]-$ benzylamine. The semicarbazide-inhibited oxidation is representative of the SSAO activity. Mean \pm SEM of the number of determinations indicated in parentheses. Analysis of variance showed that the factor tissue was highly influencing SSAO activity $\left(P<10^{-18}\right)$.

consumption occurred between the beginning and the end of the 8-week treatment, the ingestion of such dose of semicarbazide was calculated to reach a mean daily intake comprised between 123 and $150 \mathrm{mg} / \mathrm{kg} /$ day (equivalent to 1100 $1350 \mu \mathrm{mol} / \mathrm{kg} / \mathrm{d}$ ). This range is similar to the dose used in toxicology studies showing no signs of any distress [29] and is lower than the oral $\mathrm{LD}_{50}$ stated in mouse $(225 \mathrm{mg} / \mathrm{kg}$, available at http://www.carl-roth.de/jsp/en-de/sdpdf/4681e.pdf). The semicarbazide oral administration clearly limited the growth of young mice. An immediate decline was detected in the growth curve after the first week of treatment, while retardation persisted throughout the treatment (Figure 1). At the end of the treatment, the semicarbazide-drinking mice weighed $15 \%$ less than their control, since body weights were $24.7 \pm 0.6$ and $29.1 \pm 0.6 \mathrm{~g}$, respectively $(n=8, P<.001)$. The subsequent lowered body weight gain found after 8 weeks of semicarbazide ingestion $(2.4 \pm 0.5 \mathrm{~g})$ represented only one third of that observed in the control group $(6.9 \pm$ $0.7 \mathrm{~g}, n=8, P<.001)$. It was concomitant with a significant reduction in the cumulative water and food consumption, observed during the same period (equivalent to a $29 \%$ and $12 \%$ reduction, resp., not shown).

A clear reduction of adiposity was found in the semicarbazide-drinking mice, at least when comparing the mass of the epididymal, perirenal, retroperitoneal, and inguinal fat pads (Figure 2). Since the body weight was lower in the semicarbazide-treated mice, it was more convenient to compare the adiposomatic index between the two groups, that is, the percentage of the sum of dissected fat depots relative to body weight [8]. This index fell from $5.8 \pm 0.3 \%$ to $4.0 \pm 0.3 \%(n=8, P<.001)$, corresponding to a $31 \%$ reduction. The mass of the interscapular brown fat pad was also reduced by approximately $20 \%$ in semicarbazidedrinking mice: $82 \pm 4$ versus $103 \pm 6 \mathrm{mg}$ in control $(P<.01)$.

Two approaches were performed to further examine the limitation of WAT development. First, the determination of adipocyte diameter distribution clearly showed a fall in the proportion of the larger adipocytes, together with an 


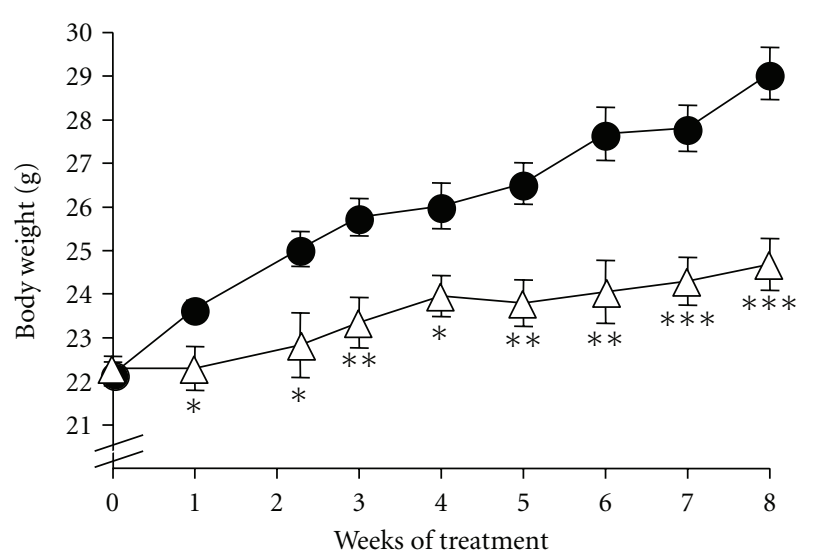

Control

$\triangle$ Semicarbazide-drinking

Figure 1: Body weight changes in mice treated or not with orally administered semicarbazide. Two groups of age- and weightmatched mice were constituted after weaning and before oral administration of semicarbazide (week 0), given at $125 \mathrm{mg} / 100 \mathrm{~mL}$ in drinking solution (semidrinking, open triangles), while control (black symbols) had free access to tap water. Mean \pm SEM of 8 determinations. Different from corresponding control at ${ }^{*} P<.05$, ${ }^{* *} P<.01,{ }^{* * *} P<.001$.

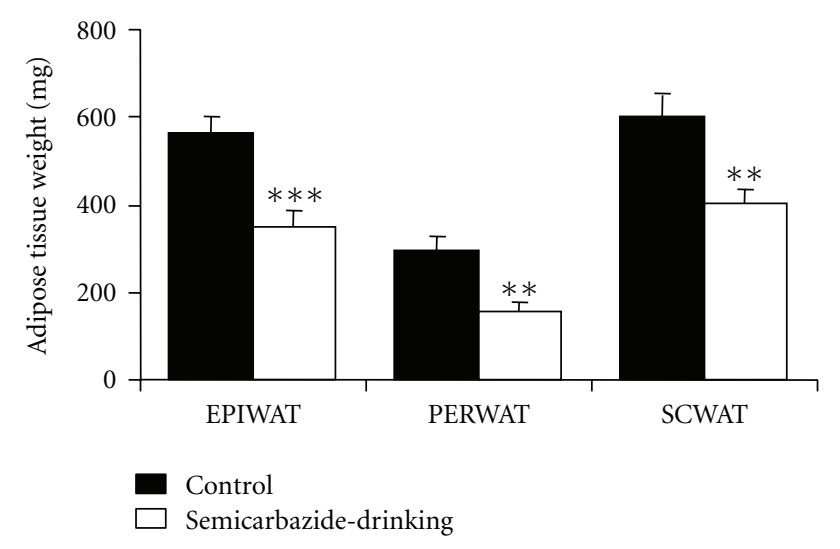

FIGURE 2: Effect of chronic semicarbazide ingestion on adipose tissue mass in male mice. Mean \pm SEM of 8 determinations of epididymal (EPIWAT), perirenal + retroperitoneal (PERWAT), or inguinal (SCWAT) fad pad weights. Different from corresponding control (black columns) at ${ }^{* *} P<.01,{ }^{* * *} P<.001$.

increase in the proportion of small fat cells (Figure 3(a)). This resulted in a significant decrease of the mean fat cell size (Figure 3(b)), reflecting an impaired lipid accumulation inside the fat cells. Second, the DNA content per $100 \mathrm{mg}$ tissue was greater in WAT from semicarbazide-drinking mice than in control, indicating that more numerous and smaller cells were present per unit of tissue mass in the treated than in the control animals, the fat depots of which contained larger, heavier, lipid-laden adipocytes (Figure 3(c)). These two approaches, together with the lack of increased adipogenic markers (see below), indicated that the WAT of

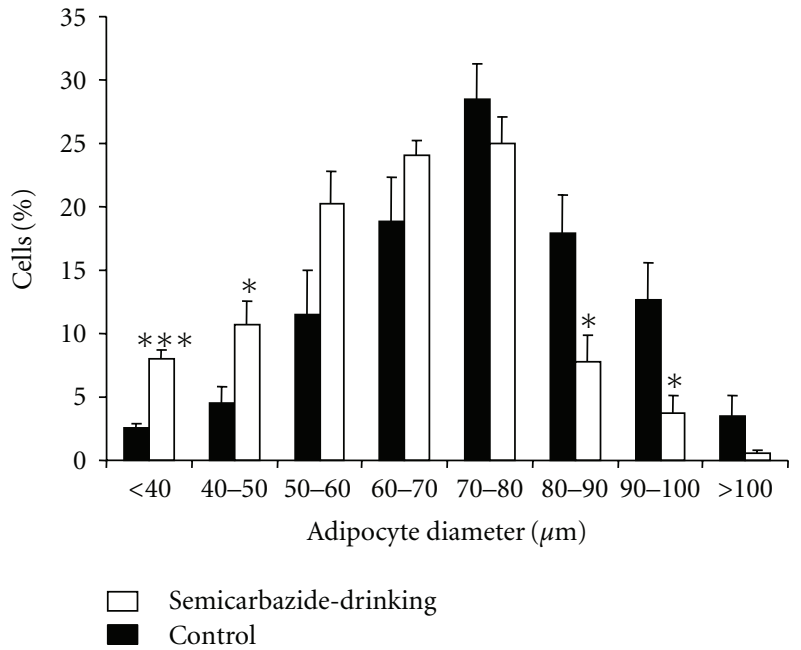

(a)

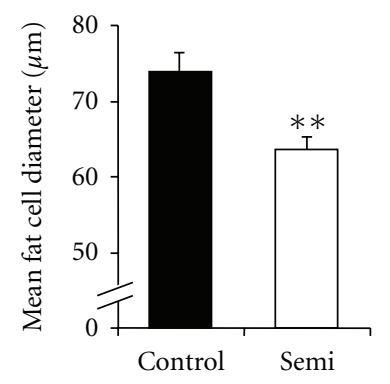

(b)

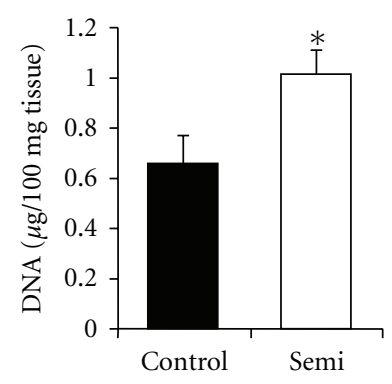

(c)
FIGURE 3: Effect of chronic semicarbazide ingestion on the cellularity of visceral adipose tissues. Adipocytes isolated from visceral adipose tissues were subjected to size analysis and counted according to the indicated distribution of cell diameter classes (a). Mean fat cell diameter was calculated (b) while DNA was extracted from pieces of visceral WAT and determined as described in Section 2 (c). Mean \pm SEM of six mice per group. Different from control at ${ }^{*} P<.05,{ }^{* *} P<.01,{ }^{* * *} P<.001$.

semicarbazide-drinking mice partially escaped to the agedependent lipid accumulation and contained adipocytes having a reduced cell size/mass when compared with agematched control mice.

Moreover, the maximal lipolytic activity of the adipocytes was elevated in the semicarbazide-treated group: in fat cells isolated from epididymal plus retroperitoneal and perirenal fat pads (pooled as visceral WAT, to obtain sufficient amount of biological material for the various determinations), the glycerol released in response to $10 \mu \mathrm{M}$ isoprenaline was 3.14 \pm 0.72 versus $1.90 \pm 0.20 \mu \mathrm{mol} / 100 \mathrm{mg}$ lipids $/ 90 \mathrm{~min}$ in control $(n=4, P<.05)$. Such observation supported that these small adipocytes were more metabolically active than larger fat cells. Therefore, chronic semicarbazide ingestion clearly appeared to prevent adipocyte hypertrophy and to facilitate lipid mobilization.

3.3. Benzylamine-Dependent Effects in Adipocytes. Of worth was to investigate whether the insulin-like effects of amines 
were abolished in fat cells after SSAO blockade. The glucose uptake was measured ex vivo in adipocytes isolated from visceral WAT. The submaximal stimulation of deoxyglucose uptake by $10 \mathrm{nM}$ insulin reached $2.9 \pm 0.3$-fold increase over basal in control adipocytes, while it was equivalent to a $4.5 \pm 0.5$-fold increase in semicarbazide-drinking mice ( $n=8, P<.01)$. Again, the smaller fat cell size of semicarbazide-treated mice likely accounted for such enhanced insulin responsiveness. On the opposite, the insulin-like effect of benzylamine was dramatically impaired in the semicarbazide-treated mice, especially when the SSAO substrate was tested at $0.1 \mathrm{mM}$ in the presence of $0.1 \mathrm{mM}$ vanadium (Figure 4). The combination of vanadium and benzylamine produced more than $80 \%$ of the maximal insulin effect in control cells, as previously reported [16], whereas this effect did not exceed $25 \%$ after semicarbazide treatment. Similarly, the weak effect of benzylamine alone on glucose uptake also exhibited a tendency to be reduced in semicarbazide-treated mice (not shown).

3.4. Amine Oxidase Activities in Adipose Tissue. It was then verified whether SSAO activity was really blocked in WAT after semicarbazide treatment. As shown in Figure 5(a), the oxidation of benzylamine was totally abolished in the subcutaneous WAT of semicarbazide-drinking mice. This was in agreement with the fact that, in control WAT, benzylamine oxidation was exclusively SSAO-dependent. On the contrary, tyramine was oxidized by SSAO and MAO as well. Its oxidation was lower in the semicarbazidetreated mice than in control. The remaining tyramine oxidation found after semicarbazide treatment was explained by an unaltered MAO-dependent oxidation together with an almost total disappearance of the SSAO component (Figure 5(b)). A similar obliteration of SSAO activity was found in visceral WAT after semicarbazide treatment: the oxidation of $0.1 \mathrm{mM}$ benzylamine fell from $2.74 \pm 0.28$ to $0.06 \pm 0.02 \mathrm{nmol} / \mathrm{mg}$ protein $/ \mathrm{min}(n=6, P<.001)$.

The total blockade of SSAO activity obtained after semicarbazide treatment was not accompanied by any alteration of MAO activity. This was supported by the lack of change in the expression of genes encoding for the monoamine oxidases A and B in adipocytes (Table 2). Similarly, there was no change for AOC2 gene, which is poorly expressed in WAT. More surprising was the decline in AOC3 expression, the gene encoding for SSAO, traducing that prolonged semicarbazide treatment did not only inhibit the SSAO enzymatic activity but also reduced its expression in WAT.

3.5. Semicarbazide-Induced Changes in Gene Expression in Adipocytes. To further analyse the changes occurring in adipose tissue after semicarbazide treatment, the expression of several genes was quantified in adipocytes from visceral WAT. As expected, the leptin expression was strongly inhibited in adipocytes from the smaller fat pads found in the semicarbazide-treated group (Table 2). However, no noticeable change in the expression of other adipokines was found, irrespective of their detrimental (TNF $\alpha$, IL-6, resistin) or beneficial (adiponectin) effect on insulin resistance, with

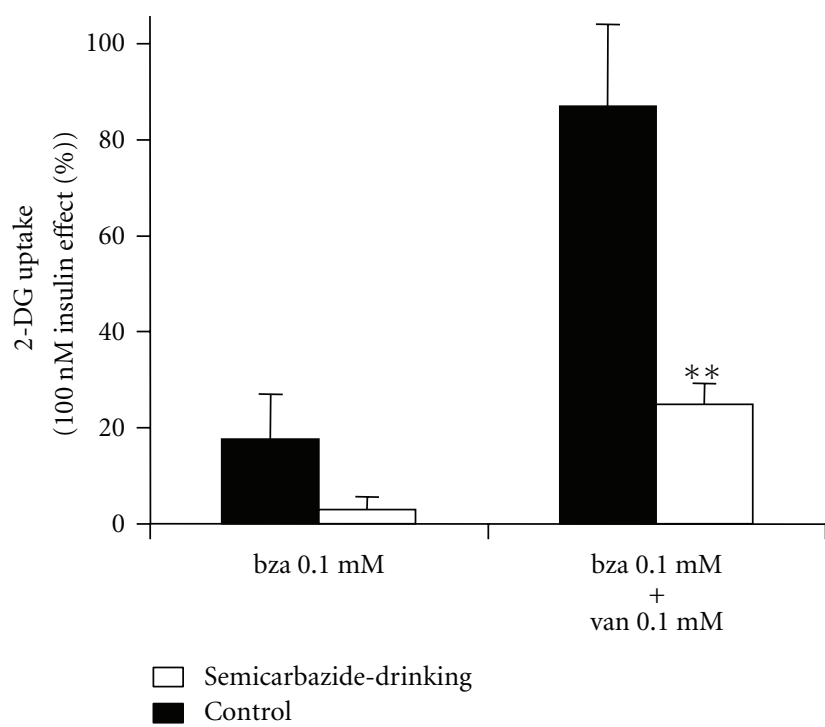

FIGURE 4: Influence of 8-week oral administration of semicarbazide on benzylamine-induced glucose transport in mouse adipocytes. Visceral adipocytes were incubated with $0.1 \mathrm{mM}$ benzylamine alone (bza) or combined with $0.1 \mathrm{mM}$ vanadate (bza + van) for 2DG uptake assay. Data are expressed as percentage of response to $100 \mathrm{nM}$ insulin, which maximally stimulated basal uptake by 3.3 \pm 0.4 -fold in control (black columns) and by $4.8 \pm 0.8$-fold in semicarbazide-drinking mice (open columns). Mean \pm SEM of 8 determinations. Different from control at ${ }^{* *} P<.01$.

the exception of apelin, the mRNA abundance of which exhibited a $50 \%$ reduction that failed to reach statistical significance $(P<.1)$. Regarding the genes involved in energy supply to adipocytes, there was no clear change in LPL or FAS expression (Table 2). The expression of the ubiquitous glucose transporter GLUT1 was reduced, but not that of the insulin-sensitive GLUT4. Catalase expression remained unaffected (not shown). Lastly, the expression of the adipogenic markers aP2 and PPAR $\gamma_{2}$ genes was unchanged in the semicarbazide-treated group (they were equivalent to $77 \%$ and $93 \%$ of the levels found in control, resp.) while a tendency to decrease was found for the macrophage marker F4/80 (not shown). Taken together, these last observations argued that the increased DNA content per mass unit of WAT was not due to an increased adipogenesis or to an infiltration of immune cells but was rather the consequence of the reduced size of the adipocytes: being of smaller mass, they were in higher number per $100 \mathrm{mg}$ tissue than in control, the WAT of which was constituted with larger fat cells.

\subsection{Absence of Abnormalities in the Circulating Metabolic Parameters of Semicarbazide-Drinking Mice. Regarding the adverse effects that might be provoked by semicarbazide prolonged ingestion, no change was found at the plasma level for the circulating values of glucose, insulin, triglycerides, free fatty acids, or cholesterol (not shown). Similarly, the plasma markers of lipid peroxidation did not differ between control and semicarbazide-drinking mice (malonyldialde- hyde equivalents were $23.9 \pm 2.0$ and $22.5 \pm 3.2$, resp., $n=8$ )}




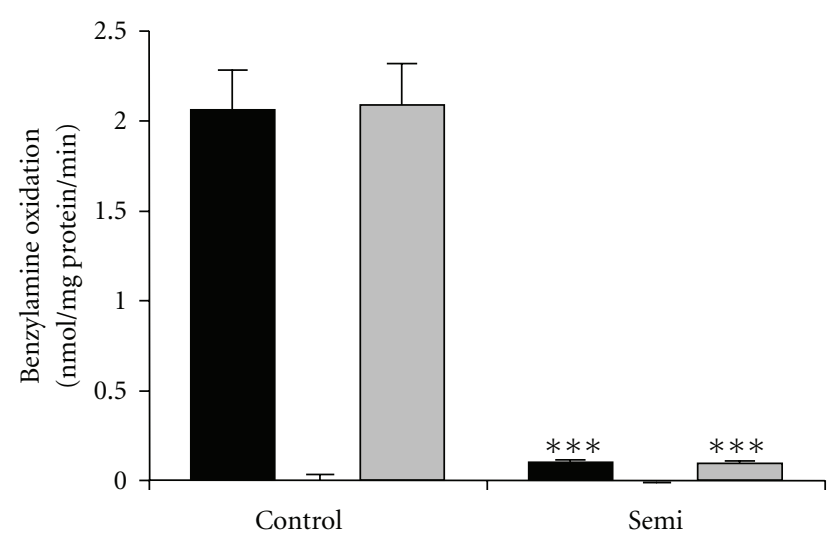

(a)

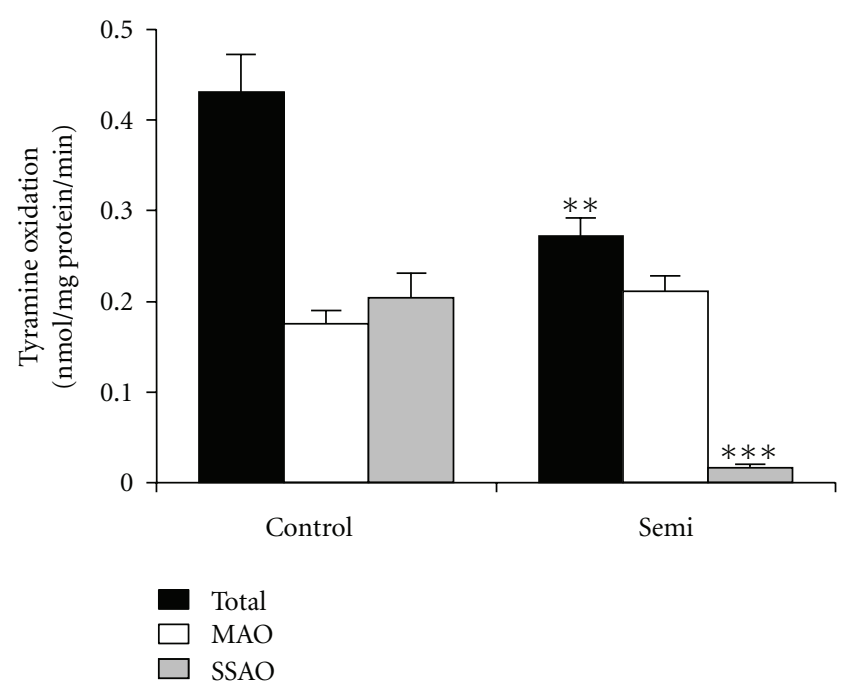

(b)

FIGURE 5: Influence of 8-week oral treatment with semicarbazide on the benzylamine (a) and tyramine (b) oxidation in subcutaneous WAT. Homogenates were preincubated for 15 minutes without (total oxidation, black columns), with $0.5 \mathrm{mM}$ pargyline (in order to determine pargyline-sensitive component as MAO, open columns), or with $1 \mathrm{mM}$ semicarbazide (to determine the semicarbazidesensitive component as SSAO, shaded columns). Then, they were incubated for 30 minutes in the presence of $0.1 \mathrm{mM}$ benzylamine (a) or $0.5 \mathrm{mM}$ tyramine (b). Mean \pm SEM of 6 determinations. Different from corresponding control at ${ }^{* *} P<.01$, ${ }^{* * *} P<.001$.

\section{Discussion}

The present study highlights decrease in both body weight gain and fattening as marked consequences of semicarbazide subchronic ingestion. The total SSAO blockade found in WAT, together with the decreased lipogenic effect of benzylamine, which can be considered as one representative of the dietary amines reaching visceral fat during the digestion process, led us to propose that pharmacological inhibition of SSAO can be considered as a way to mitigate obesity. Since SSAO is largely expressed in fat depots, a peripheral mode of action can be envisaged, but other central mechanisms,
TAble 2: Gene expression in visceral adipocytes from semicarbazide-drinking or control mice.

\begin{tabular}{lcc}
\hline Gene & Control & Semicarbazide-treated \\
\hline Amine oxidases: & & \\
MAO-A & $66.2 \pm 22.3$ & $35.9 \pm 11.7$ \\
MAO-B & $154 \pm 21$ & $133 \pm 31$ \\
AOC2 & $2.0 \pm 0.4$ & $1.5 \pm 0.2$ \\
AOC3 & $4750 \pm 555$ & $2420 \pm 275^{* *}$ \\
Adipokines: & & \\
Adiponectin & $22568 \pm 1942$ & $19068 \pm 2195$ \\
Resistin & $3837 \pm 300$ & $4143 \pm 374$ \\
leptin & $2068 \pm 365$ & $853 \pm 80^{* *}$ \\
IL-6 & $792 \pm 230$ & $718 \pm 198$ \\
TNF-alpha & $16.9 \pm 2.6$ & $23.5 \pm 8.1$ \\
Apelin & $26.0 \pm 9.3$ & $12.5 \pm 4.3$ \\
Fuel metabolism: & & \\
GLUT1 & $187 \pm 28$ & $103 \pm 18^{*}$ \\
GLUT4 & $440 \pm 38$ & $429 \pm 51$ \\
LPL & $47742 \pm 3860$ & $39274 \pm 3152$ \\
\hline
\end{tabular}

mRNA abundance of the indicated genes was determined by rt-PCR and expressed using the arbitrary units detailed in Section 2. Mean \pm SEM of 8 determinations. Different from control at $* P<.05$; ${ }^{* *} P<.01$.

participating to the observed $12 \%$ reduction of calorie intake cannot be excluded.

The clear-cut blockade of benzylamine oxidation found in the WAT of treated mice agrees with the fact that benzylamine oxidation is almost exclusively catalyzed by SSAO activity in this tissue. As a consequence, the insulinlike action of the SSAO substrate benzylamine on glucose transport was abolished in adipocytes from semicarbazidetreated mice. This was concomitant with substantial reductions in the fat mass and in the mean fat cell size. Whether the decrease in SSAO expression was a consequence of fat cell size reduction appears unlikely since the shrinking of lipid stores observed during prolonged fasting is not accompanied by a reduction of SSAO activity in adipose depots [28]. On the opposite, agents known to inhibit SSAO have been observed to limit lipogenesis in vitro [20]. On the basis of these previous observations, it can be proposed that inhibition of lipogenic effects of endogenous/dietary SSAO substrates participates to the limitation of fat deposition. The reduced number of large adipocytes together with the increased DNA content per WAT mass and the unchanged adipocyte differentiation markers $\left(\mathrm{aP} 2, \operatorname{PPAR} \gamma_{2}\right)$, indicated that the differentiated adipocytes already present at the beginning of treatment, at the age of 5 weeks, were less capable to accumulate lipids in semicarbazide-drinking mice than in the control group. The resulting fat cells of moderate size were present in a larger number per mass unit and consequently gave higher DNA richness per mass unit of WAT, higher lipolytic capacity, and higher insulin responsiveness. In this view, the inhibition of peripheral SSAO, which is abundant in fat cells $[15,25]$, but which is not expressed in neurones, may represent a fascinating novel 
approach to modulate WAT development. Such paradigm is relevant only whether endogenous or dietary amines spontaneously exert insulin-like effects, which is far from being demonstrated. Indeed, it is widely accepted that numerous amines are spontaneously present in foods $[30,31]$ or may be generated during digestion process by the intestinal flora [32]: for instance, tyramine is present at around $45 \mathrm{mg} / \mathrm{kg}$ in rodent chow [33] and benzylamine reaches around $3-10 \mathrm{mg} / \mathrm{kg}$ in edible vegetables [34]. However, it has never been demonstrated (or ruled out) whether such dietary amines might exert in vivo the lipogenic effects they exhibit in vitro [19], and so facilitate fat accretion once ingested.

Moreover, SSAO is not only expressed in fat cells but also in vessels, in endothelial cells, where it is involved in leukocyte extravasation, and in smooth muscle cells, where it is involved in glucose transport [11] and in collagen-elastin maturation $[2,3]$. The tendency to decrease the F4/80 macrophage marker level found in mice after semicarbazide exposure is probably related to the antiinflammatory properties of SSAO/VAP-1 blocking agents [35]. Whether the improved in vitro responsiveness to insulin in the adipocytes from semicarbazide-drinking mice is a consequence of such reduced low-grade inflammation or is related to the smaller size of fat cells remains to be determined. In the treated adipocytes, there was no change in the expression of TNF $\alpha$ and IL-6, two cytokines involved in insulin resistance, together with a decrease of the proinflammatory adipokine leptin and a tendency to lower the expression of apelin, another adipokine recently shown to facilitate glucose utilization [36]. Such absence of enhanced WAT inflammation was associated with an absence of oxidative stress (evidenced by unchanged lipid peroxidation markers or catalase content). This context may explain the increased ex vivo insulin responsiveness of adipocytes, which was not sufficient to fill the fat cells of semicarbazide-drinking mice via sustained lipogenic pathways, since it was occurring together with a reduced calorie intake. Of interest is to test whether the insulinsensitizing action of SSAO blockade also occurs acutely in obese insulin-resistant models, and improve the insulindependent and independent antilipolytic responses, since adipose tissue lipolysis regulation has been proposed to be instrumental in the treatment of obesity and metabolic syndrome [37].

The major concern is that semicarbazide itself cannot currently be proposed as antiobesity drug since this derivative has been described to exert deleterious toxic effects. In 2003, the European Food Safety Authority has banned the use of azodicarbonamide as a blowing agent for plastics used in food processing or packaging, or as a flour treatment, since it can be transformed into semicarbazide, which is suspected to be a food contaminant exerting toxicological effects on consumers. However, this hydrazine derivative is not only a food contaminant, the origin of which is limited to the transformation of known chemical substances used (or even prohibited) in food processing [23], but can also naturally occur in certain foods $[38,39]$. As a consequence, the DNA damaging effects of ingested semicarbazide have been intensively studied to bring health risk assessments. But these recent toxicological studies inconsistently demonstrated the genotoxic properties of semicarbazide, since they raised variations according to the model and the protocol used. Briefly, semicarbazide has very poor genotoxic effects on human lymphocytes when tested in vitro [23] and is not genotoxic in mice when administered in vivo at $120 \mathrm{mg} / \mathrm{kg}$ b.w. [29]. However semicarbazide exhibits carcinogenic potentialwith marginal statistical relevance-when orally given to rats at $150 \mathrm{mg} / \mathrm{kg}$ [23]. Of note, other aspects of semicarbazide toxicity have also been stated very recently and have produced more consensual results. Indeed, a toxicological study showed a dramatic decrease in body weight with orally given semicarbazide as mixed in the food at doses up to $1000 \mathrm{ppm}$, giving a daily intake of 65 to $70 \mathrm{mg} / \mathrm{kg}$ in male and female rats [22]. The decrease in adiposity was not assessed in this study. However, the relative weights of the brain, heart, and kidneys were unchanged or even increased, depending on the sex, while body weights were clearly lower in the semicarbazidetreated rats. This may indicate that while semicarbazide ingestion limits WAT expansion, as assessed in our conditions, other organs are preserved during the body weight loss. Unfortunately, exposure to semicarbazide was accompanied by serious adverse effects such as deformation of bones and lesions of articular cartilages [22]. In the aorta, alteration of connective tissues was suspected to increase fragility of arterial walls. Nonetheless, no inflammatory disease was found. In view of these toxicological effects of chronic exposure to semicarbazide, the no observed adverse effect level (NOAEL) was estimated by the authors to be lower than $18-21 \mathrm{mg} / \mathrm{kg} / \mathrm{day}$ in rat. Accordingly, the NOAEL in juvenile rats on another strain has recently been proposed to be lower than $40 \mathrm{mg} / \mathrm{kg}$ for semicarbazide oral administration [21]. In this study describing the toxicity of semicarbazide on biochemical and behavioural parameters, the authors observed a clear-cut inhibition of body weight gain and defects in cartilage mineralization [21]. During the completion of the present work, the same italian group reported that semicarbazide behaves as an endocrine disrupter, being able to reduce estrogen levels or to alter testosterone catabolism [40]. Anyhow, semicarbazide inhibited body weight gain and food consumption in postweaning male and female rats when orally given from the dose of 40 to $140 \mathrm{mg} / \mathrm{kg} / \mathrm{d}$ period.

Thus, semicarbazide administration is univocally recognized to reduce body weight gain in diverse obese or nonobese models $[2,3,7,8,21]$ and regarding this aspect our findings are confirmatory. Our detailed analysis of adiposity of juvenile mice, together with the high expression of SSAO in adipocytes, brings novel insight, focusing the attention on WAT as a major target of semicarbazide. However, this hydrazine derivative cannot be safely used as a "slimming" agent for toxic issues in bones, joints, and vessels. Although other SSAO inhibitors have also been reported to limit body weight gain, several semicarbazide effects other than inhibiting SSAO will be discussed here in an attempt to detect whether SSAO blockade is necessary and/or sufficient to limit fat deposition.

Another well-recognized effect of semicarbazide is to inhibit lysyl oxidase, a copper-containing amine oxidase 
involved in the stabilization of extracellular matrix by crosslinking of proteins such as collagen and elastin [2, 3], therefore explaining part of the semicarbazide toxic effects on bones and joints. However, the lysyl oxidase inhibitor $\beta$-aminopropionitrile, reported to decrease insoluble elastin and collagen content and to impair collagen cross-linking does not decrease body weight gain, excepted when combined with semicarbazide [3].

Semicarbazide has been reported to inhibit glutamic acid decarboxylase, a $\gamma$-amino butyric acid-synthesizing enzyme $[41,42]$, and its administration is therefore believed to impair $\gamma$-amino butyric acid (GABA) formation and to lower GABA brain levels. This has been proven to induce "freezing" behaviour in rodents after central administration [43]. However, it is striking to note that relatively high doses of gammavinyl GABA, an irreversible inhibitor of the enzyme GABAtransaminase, which is involved GABA catabolic pathway, have been recently reported to produce significant weight loss in rats [44]. Likewise, another inhibitor of the GABA degradation exhibits anorectic potency in obese rats [45], while it has been proposed that a rat strain resistant to highfat diet feeding reduces its food intake via an elevation of brain GABA levels [46]. In fact, it is difficult to understand how the chronic in vivo administration of semicarbazide, supposed to inhibit GABA synthesis, alters the GABA-ergic systems in a manner that results in reduced food intake, as it is the case with inhibitors of GABA degradation. To pour more complexity, it has been recently reported that several semicarbazide derivatives elevate GABA levels in the midbrain and exert anticonvulsant activity [47]. It could be therefore of interest to determine the relative proportion of the central GABA-ergic alterations and of the peripheral SSAO blockade in the "slimming" action of semicarbazide before establishing whether more selective SSAO blockers, devoid of interaction with the GABA-ergic system, also elicit antiobesity effects. Otherwise, the current number of SSAOinteracting agents that have been also reported to reduce weight gain (FPFA [1], aminoguanidine [6], phenelzine [8]) suggests a nonnegligible influence of the component related to SSAO inhibition in the observed effect of semicarbazide.

Semicarbazide has also been described to inhibit sphingosine-1-phosphate (S1P) lyase [48], but it is not clear whether such nonspecific inhibition may have some bearing on our observations. As in the case of GABAergic system, it must be quoted that novel semicarbazide derivatives act differently from semicarbazide itself, since instead of inhibiting a S1P-degrading enzyme, they are selective inhibitors of a subset of the G protein-coupled S1P receptors (S1P2) [49]. This emphasizes that semicarbazide is a molecule with multiple aspects, each one being selectively developed by the association of a functional and selective moiety to the semicarbazide turntable structure. Such drug design is out of the scope of the present study, while SSAO inhibition, obtained by the various above-mentioned pharmacological agents was always found to be concomitant with a decreased body weight gain. The need for searching novel, selective SSAO inhibitors is not only a concern for our proposed antiobesity pharmacology, but also relies with the general biology of the enzyme, which will soon change of denomination, from SSAO to primary amine oxidase, a fact indicating that its definition based on its sensitivity to semicarbazide will become historical once the many other inhibitors under development will be fully characterized.

\section{Conclusions}

Taken together, our results demonstrate that oral administration of the prototypical SSAO inhibitor semicarbazide limits weight gain and fat deposition in mouse. In spite of the toxicity of the high doses of this agent, the use of other specific SSAO inhibitors might reveal more beneficial for the pharmacologic treatment of obesity.

\section{Conflict of Interests}

The authors have no conflict of interests to declare.

\section{Acknowledgments}

This study was partly supported by a grant from INTERREG IVB-SUDOE-FEDER (DIOMED, SOE1/P1/E178, http://diomed-sudoe.eu), by "Communauté de Travail des Pyrénées" and by "Balaton Programme" for French-Spanish and French-Hungarian exchanges, respectively. The authors express gratitude to all the members of Inserm U1048 team 3 and of IFR 150 (Toulouse) for invaluable help.

\section{References}

[1] P. H. Yu, M. Wang, H. Fan, Y. Deng, and D. GubisneHaberle, "Involvement of SSAO-mediated deamination in adipose glucose transport and weight gain in obese diabetic KKAy mice," American Journal of Physiology, vol. 286, no. 4, pp. E634-E641, 2004.

[2] N. Mercier, K. El Hadri, M. Osborne-Pellegrin et al., "Modifications of arterial phenotype in response to amine oxidase inhibition by semicarbazide," Hypertension, vol. 50, no. 1, pp. 234-241, 2007.

[3] N. Mercier, A. Kakou, P. Challande, P. Lacolley, and M. Osborne-Pellegrin, "Comparison of the effects of semicarbazide and $\beta$-aminopropionitrile on the arterial extracellular matrix in the Brown Norway rat," Toxicology and Applied Pharmacology, vol. 239, no. 3, pp. 258-267, 2009.

[4] P. H. Yu and D. M. Zuo, "Aminoguanidine inhibits semicarbazide-sensitive amine oxidase activity: implications for advanced glycation and diabetic complications," Diabetologia, vol. 40, no. 11, pp. 1243-1250, 1997.

[5] B. O. Nilsson, "Biological effects of aminoguanidine: an update," Inflammation Research, vol. 48, no. 10, pp. 509-515, 1999.

[6] D. Prévot, Z. Soltesz, V. Abello et al., "Prolonged treatment with aminoguanidine strongly inhibits adipocyte semicarbazide-sensitive amine oxidase and slightly reduces fat deposition in obese Zucker rats," Pharmacological Research, vol. 56, no. 1, pp. 70-79, 2007.

[7] C. Carpéné, Z. Iffiú-Soltesz, S. Bour, D. Prévot, and P. Valet, "Reduction of fat deposition by combined inhibition of monoamine oxidases and semicarbazide-sensitive amine oxidases in obese Zucker rats," Pharmacological Research, vol. 56, no. 6, pp. 522-530, 2007. 
[8] C. Carpéné, V. Abello, Z. Iffiú-Soltész, N. Mercier, B. Fève, and P. Valet, "Limitation of adipose tissue enlargement in rats chronically treated with semicarbazide-sensitive amine oxidase and monoamine oxidase inhibitors," Pharmacological Research, vol. 57, no. 6, pp. 426-434, 2008.

[9] J. O’Sullivan, M. Unzeta, J. Healy, M. I. O’Sullivan, G. Davey, and K. F. Tipton, "Semicarbazide-sensitive amine oxidases: enzymes with quite a lot to do," NeuroToxicology, vol. 25, no. 1-2, pp. 303-315, 2004.

[10] S. Jalkanen, M. Karikoski, N. Mercier et al., "The oxidase activity of vascular adhesion protein-1 (VAP-1) induces endothelial E- and P-selectins and leukocyte binding," Blood, vol. 110, no. 6, pp. 1864-1870, 2007.

[11] K. El Hadri, M. Moldes, N. Mercier, M. Andreani, J. Pairault, and B. Feve, "Semicarbazide-sensitive amine oxidase in vascular smooth muscle cells: differentiation-dependent expression and role in glucose uptake," Arteriosclerosis, Thrombosis, and Vascular Biology, vol. 22, no. 1, pp. 89-94, 2002.

[12] L. Conforti, L. Raimondi, and G. A. Lyles, "Metabolism of methylamine by semicarbazide-sensitive amine oxidase in white and brown adipose tissue of the rat," Biochemical Pharmacology, vol. 46, no. 4, pp. 603-607, 1993.

[13] Y. Ochiai, K. Itoh, E. Sakurai, and Y. Tanaka, "Molecular cloning and characterization of rat semicarbazide-sensitive amine oxidase," Biological and Pharmaceutical Bulletin, vol. 28, no. 3, pp. 413-418, 2005.

[14] T. Valente, M. Solé, and M. Unzeta, "SSAO/VAP-1 protein expression during mouse embryonic development," Developmental Dynamics, vol. 237, no. 9, pp. 2585-2593, 2008.

[15] N. Morin, J. M. Lizcano, E. Fontana et al., "Semicarbazidesensitive amine oxidase substrates stimulate glucose transport and inhibit lipolysis in human adipocytes," Journal of Pharmacology and Experimental Therapeutics, vol. 297, no. 2, pp. 563-572, 2001.

[16] Z. Iffiú-Soltész, E. Wanecq, A. Lomba et al., "Chronic benzylamine administration in the drinking water improves glucose tolerance, reduces body weight gain and circulating cholesterol in high-fat diet-fed mice," Pharmacological Research, vol. 61, no. 4, pp. 355-363, 2010.

[17] E. Fontana, J. Boucher, L. Marti et al., "Amine oxidase substrates mimic several of the insulin effects on adipocyte differentiation in 3T3 F442A cells," Biochemical Journal, vol. 356, no. 3, pp. 769-777, 2001.

[18] N. Mercier, M. Moldes, K. El Hadri, and B. Fève, "Semicarbazide-sensitive amine oxidase activation promotes adipose conversion of 3T3-L1 cells," Biochemical Journal, vol. 358, no. 2, pp. 335-342, 2001.

[19] C. Carpéné, D. Daviaud, J. Boucher et al., "Short- and long-term insulin-like effects of monoamine oxidases and semicarbazide-sensitive amine oxidase substrates in cultured adipocytes," Metabolism, vol. 55, no. 10, pp. 1397-1405, 2006.

[20] F. Chiche, M. Le Guillou, G. Chétrite et al., "Antidepressant phenelzine alters differentiation of cultured human and mouse preadipocytes," Molecular Pharmacology, vol. 75, no. 5, pp. 1052-1061, 2009.

[21] F. Maranghi, R. Tassinari, V. Lagatta et al., "Effects of the food contaminant semicarbazide following oral administration in juvenile Sprague-Dawley rats," Food and Chemical Toxicology, vol. 47, no. 2, pp. 472-479, 2009.

[22] M. Takahashi, M. Yoshida, K. Inoue, T. Morikawa, and A. Nishikawa, "A ninety-day toxicity study of semicarbazide hydrochloride in Wistar Hannover GALAS rats," Food and Chemical Toxicology, vol. 47, no. 10, pp. 2490-2498, 2009.
[23] D. Vlastos, H. Moshou, and K. Epeoglou, "Evaluation of genotoxic effects of semicarbazide on cultured human lymphocytes and rat bone marrow," Food and Chemical Toxicology, vol. 48, no. 1, pp. 209-214, 2010.

[24] V. Visentin, S. Bour, J. Boucher et al., "Glucose handling in streptozotocin-induced diabetic rats is improved by tyramine but not by the amine oxidase inhibitor semicarbazide," European Journal of Pharmacology, vol. 522, no. 1-3, pp. 139146, 2005.

[25] S. Bour, D. Daviaud, S. Gres et al., "Adipogenesis-related increase of semicarbazide-sensitive amine oxidase and monoamine oxidase in human adipocytes," Biochimie, vol. 89, no. 8, pp. 916-925, 2007.

[26] J. Boucher, I. Castan-Laurell, D. Daviaud et al., "Adipokine expression profile in adipocytes of different mouse models of obesity," Hormone and Metabolic Research, vol. 37, no. 12, pp. 761-767, 2005.

[27] S. L. Lay, S. Krief, C. Farnier et al., "Cholesterol, a cell sizedependent signal that regulates glucose metabolism and gene expression in adipocytes," Journal of Biological Chemistry, vol. 276, no. 20, pp. 16904-16910, 2001.

[28] Z. Iffiú-Soltész, D. Prévot, and C. Carpéné, "Influence of prolonged fasting on monoamine oxidase and semicarbazidesensitive amine oxidase activities in rat white adipose tissue," Journal of Physiology and Biochemistry, vol. 65, no. 1, pp. 1124, 2009.

[29] L. Abramsson-Zetterberg and K. Svensson, "Semicarbazide is not genotoxic in the flow cytometry-based micronucleus assay in vivo," Toxicology Letters, vol. 155, no. 2, pp. 211-217, 2005.

[30] B. Pfundstein, A. R. Tricker, E. Theobald, B. Spiegelhalder, and R. Preussmann, "Mean daily intake of primary and secondary amines from foods and beverages in West Germany in 19891990," Food and Chemical Toxicology, vol. 29, no. 11, pp. 733739, 1991.

[31] B. J. McCabe-Sellers, C. G. Staggs, and M. L. Bogle, "Tyramine in foods and monoamine oxidase inhibitor drugs: a crossroad where medicine, nutrition, pharmacy, and food industry converge," Journal of Food Composition and Analysis, vol. 19, pp. S58-S65, 2006.

[32] J. M. Gabastou, L. Nugon-Baudon, Y. Robert et al., "Digestives amines of bacterial origin and behaviour. A case report," Pathologie Biologie, vol. 44, no. 4, pp. 275-281, 1996.

[33] V. Visentin, P. Marq, S. Bour et al., "Effect of prolonged treatment with tyramine on glucose tolerance in streptozotocininduced diabetic rats," Journal of Physiology and Biochemistry, vol. 59, no. 3, pp. 225-232, 2003.

[34] G. B. Neurath, M. Duenger, and F. G. Pein, "Primary and secondary amines in the human environment," Food and Cosmetics Toxicology, vol. 15, no. 4, pp. 275-282, 1977.

[35] K. Noda, S. Nakao, S. Zandi, V. Engelstädter, Y. Mashima, and A. Hafezi-Moghadam, "Vascular adhesion protein-1 regulates leukocyte transmigration rate in the retina during diabetes," Experimental Eye Research, vol. 89, no. 5, pp. 774-781, 2009.

[36] C. Dray, C. Knauf, D. Daviaud et al., "Apelin stimulates glucose utilization in normal and obese insulin-resistant mice," Cell Metabolism, vol. 8, no. 5, pp. 437-445, 2008.

[37] D. Langin, "Adipose tissue lipolysis as a metabolic pathway to define pharmacological strategies against obesity and the metabolic syndrome," Pharmacological Research, vol. 53, no. 6, pp. 482-491, 2006.

[38] K. Hoenicke, R. Gatermann, L. Hartig, M. Mandix, and S. Otte, "Formation of semicarbazide (SEM) in food by 
hypochlorite treatment: is SEM a specific marker for nitrofurazone abuse?" Food Additives and Contaminants, vol. 21, no. 6, pp. 526-537, 2004.

[39] J. G. Bendall, "Semicarbazide is non-specific as a marker metabolite to reveal nitrofurazone abuse as it can form under Hofmann conditions," Food Additives and Contaminants Part A, vol. 26, no. 1, pp. 47-56, 2009.

[40] F. Maranghi, R. Tassinari, D. Marcoccia et al., "The food contaminant semicarbazide acts as an endocrine disrupter: evidence from an integrated in vivo/in vitro approach," Chemico-Biological Interactions, vol. 183, no. 1, pp. 40-48, 2010.

[41] S. Matsushima, S. Hori, and M. Matsuda, "Conversion of 4-aminobutyraldehyde to $\gamma$-aminobutyric acid in striatum treated with semicarbazide and kainic acid," Neurochemical Research, vol. 11, no. 9, pp. 1313-1319, 1986.

[42] K. G. Borelli, C. Ferreira-Netto, and M. L. Brandão, "Distribution of Fos immunoreactivity in the rat brain after freezing or escape elicited by inhibition of glutamic acid decarboxylase or antagonism of GABA-A receptors in the inferior colliculus," Behavioural Brain Research, vol. 170, no. 1, pp. 84-93, 2006.

[43] J. M. Zanoveli and M. L. Brandão, "The dorsal periaqueductal and basolateral amygdala are necessary for the expression of conditioned place avoidance induced by semicarbazide stimulation of the dorsal periaqueductal region," Progress in Neuro-Psychopharmacology and Biological Psychiatry, vol. 32, no. 7, pp. 1715-1721, 2008.

[44] A. DeMarco, R. M. Dalal, M. Kahanda et al., "Subchronic racemic gamma vinyl-GABA produces weight loss in Sprague Dawley and Zucker fatty rats," Synapse, vol. 62, no. 11, pp. 870-872, 2008.

[45] D. V. Coscina and J. N. Nobrega, "Anorectic potency of inhibiting GABA transaminase in brain: studies of hypothalamic, dietary and genetic obesities," International Journal of Obesity, vol. 8, no. 1, pp. 191-200, 1984.

[46] J. S. Fisler, H. Shimizu, and G. A. Bray, "Brain 3-hydroxybutyrate, glutamate, and GABA in a rat model of dietary obesity," Physiology and Behavior, vol. 45, no. 3, pp. 571-577, 1989.

[47] E. Jain, Y. Kumar, J. Stables, and R. Sinha, "Menthone semicarbazides and thiosemicarbazides as anticonvulsant agents," Medicinal Chemistry, vol. 6, no. 1, pp. 44-50, 2010.

[48] P. Bandhuvula, H. Fyrst, and J. D. Saba, "A rapid fluorescence assay for sphingosine-1-phosphate lyase enzyme activity," Journal of Lipid Research, vol. 48, no. 12, pp. 2769-2778, 2007.

[49] A. Murakami, H. Takasugi, S. Ohnuma et al., "Sphingosine 1phosphate (S1P) regulates vascular contraction via S1P receptor: investigation based on a new S1P receptor antagonist," Molecular Pharmacology, vol. 77, no. 4, pp. 704-713, 2010. 


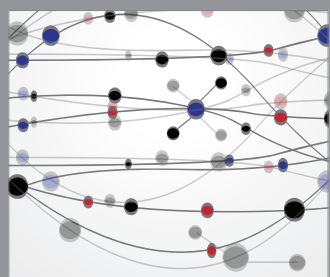

The Scientific World Journal
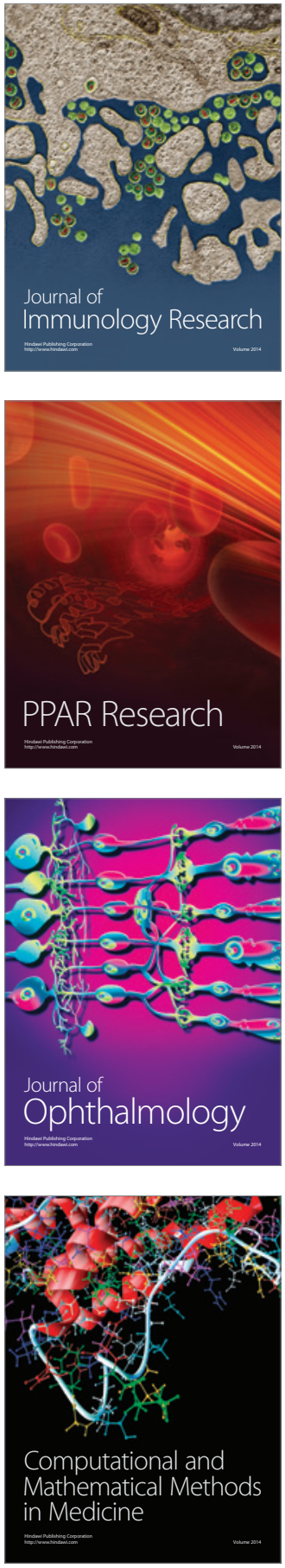

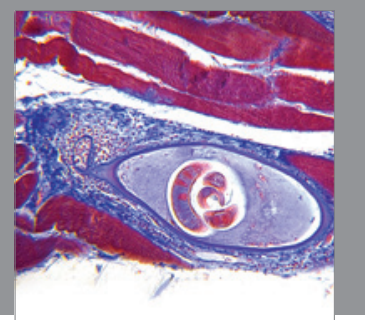

Gastroenterology

Research and Practice
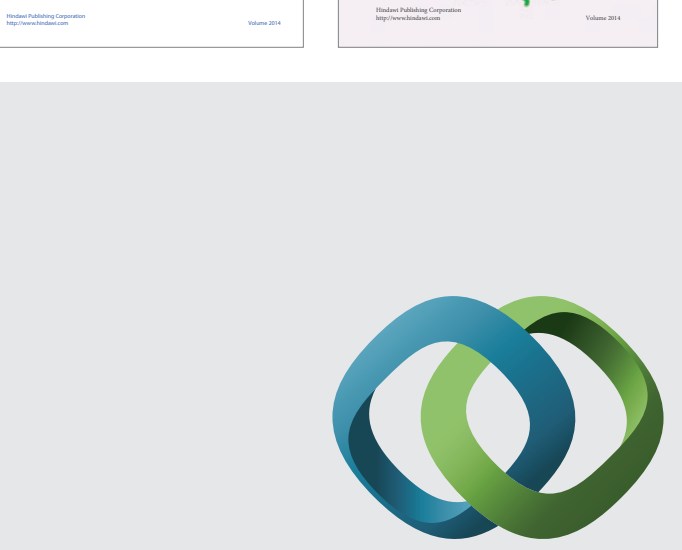

\section{Hindawi}

Submit your manuscripts at

http://www.hindawi.com
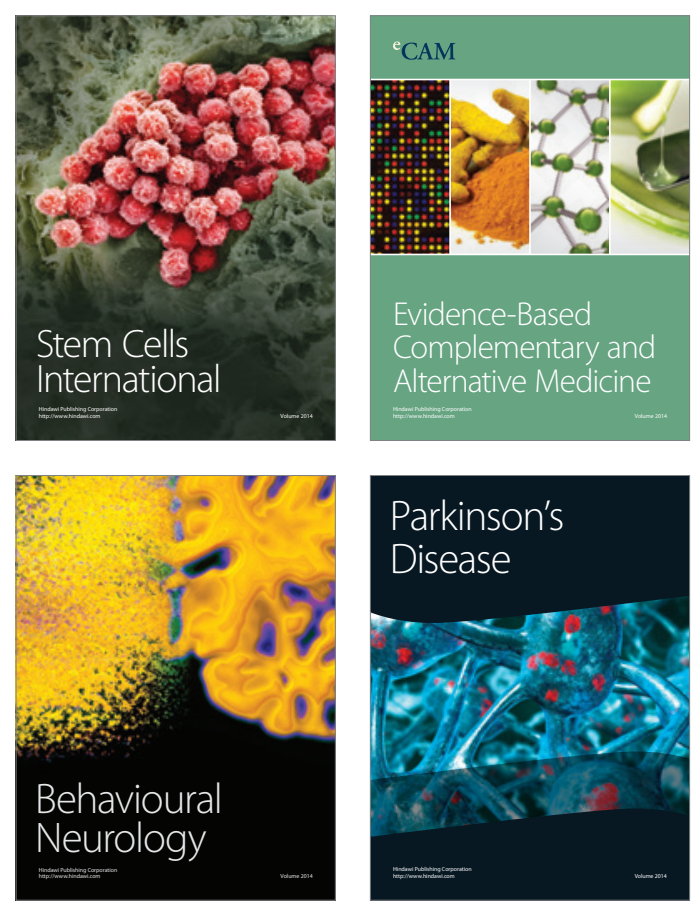

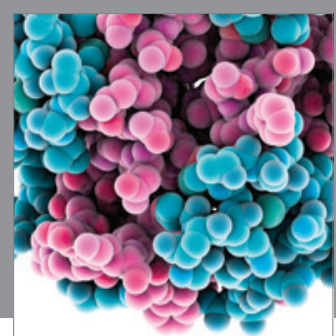

Journal of
Diabetes Research

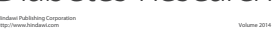

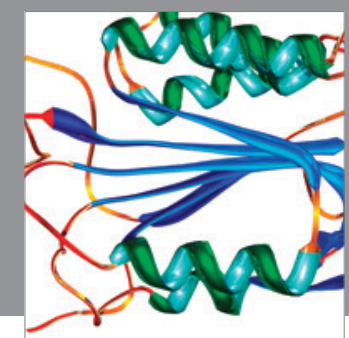

Disease Markers
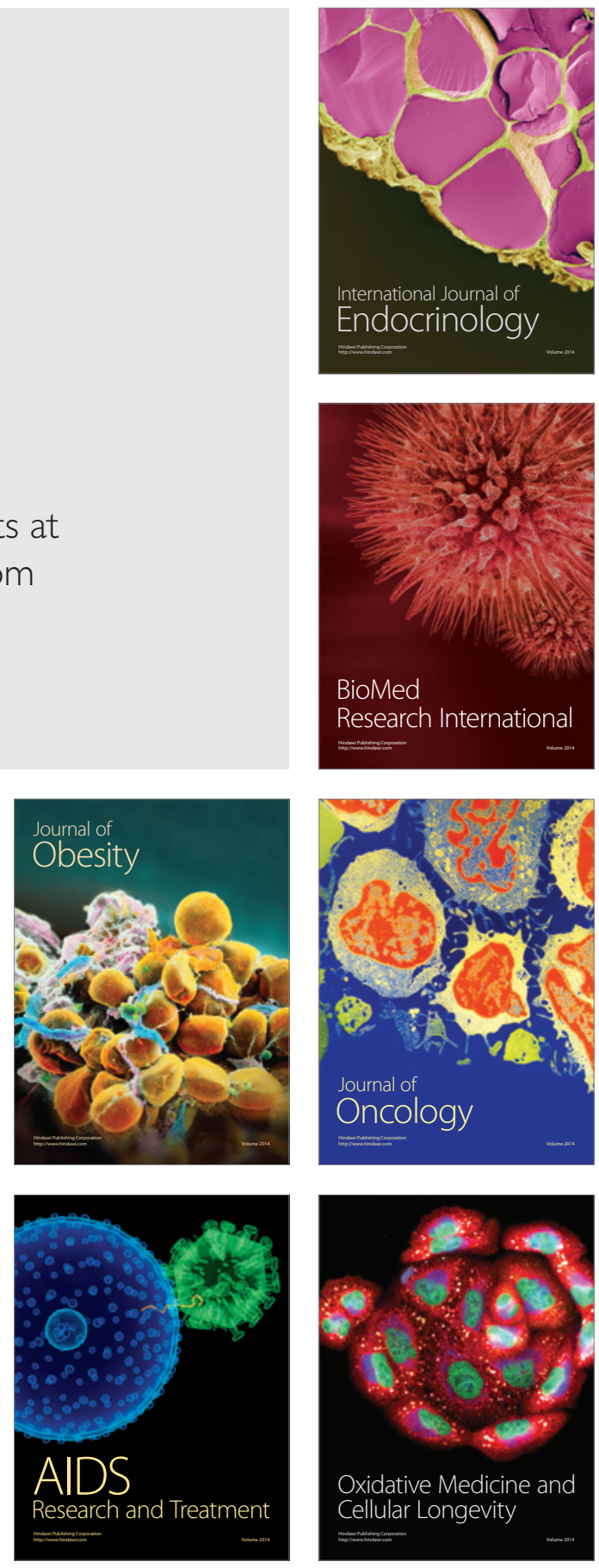